\title{
Biogenic Synthesis of Copper Nanoparticles Using Bacterial Strains Isolated from an Antarctic Consortium Associated to a Psychrophilic Marine Ciliate: Characterization and Potential Application as Antimicrobial Agents
}

\author{
Maria Sindhura John ${ }^{1}$, Joseph Amruthraj Nagoth ${ }^{1}{ }^{\mathbb{D}}$, Marco Zannotti $^{2, * \mathbb{D}}$, Rita Giovannetti ${ }^{2} \mathbb{D}$, Alessio Mancini $^{1}$, \\ Kesava Priyan Ramasamy ${ }^{1}$, Cristina Miceli ${ }^{1}$ (D) and Sandra Pucciarelli ${ }^{1, * \mathbb{D}}$ \\ 1 School of Biosciences and Veterinary Medicine, Biosciences and Biotechnology Division, \\ University of Camerino, 62032 Camerino, Italy; mariasindhura.john@unicam.it (M.S.J.); \\ josephamruthraj.nagoth@unicam.it (J.A.N.); alessio.mancini@unicam.it (A.M.); \\ kesava.ramasamy@unicam.it (K.P.R.); cristina.miceli@unicam.it (C.M.) \\ 2 School of Sciences and Technology, Chemistry Division, University of Camerino, 62032 Camerino, Italy; \\ rita.giovannetti@unicam.it \\ * Correspondence: marco.zannotti@unicam.it (M.Z.); sandra.pucciarelli@unicam.it (S.P.)
}

check for updates

Citation: John, M.S.; Nagoth, J.A.; Zannotti, M.; Giovannetti, R.; Mancini, A.; Ramasamy, K.P.; Miceli, C.; Pucciarelli, S. Biogenic Synthesis of Copper Nanoparticles Using Bacterial Strains Isolated from an Antarctic Consortium Associated to a Psychrophilic Marine Ciliate: Characterization and Potential Application as Antimicrobial Agents. Mar. Drugs 2021, 19, 263. https:// doi.org/10.3390/md19050263

Academic Editor: Daniela Giordano

Received: 31 March 2021

Accepted: 4 May 2021

Published: 8 May 2021

Publisher's Note: MDPI stays neutral with regard to jurisdictional claims in published maps and institutional affiliations.

Copyright: (c) 2021 by the authors. Licensee MDPI, Basel, Switzerland. This article is an open access article distributed under the terms and conditions of the Creative Commons Attribution (CC BY) license (https:/ / creativecommons.org/licenses/by/ $4.0 /)$.

\begin{abstract}
In the last decade, metal nanoparticles (NPs) have gained significant interest in the field of biotechnology due to their unique physiochemical properties and potential uses in a wide range of applications. Metal NP synthesis using microorganisms has emerged as an eco-friendly, clean, and viable strategy alternative to chemical and physical approaches. Herein, an original and efficient route for the microbial synthesis of copper NPs using bacterial strains newly isolated from an Antarctic consortium is described. UV-visible spectra of the NPs showed a maximum absorbance in the range of 380-385 nm. Transmission electron microscopy analysis showed that these NPs are all monodispersed, spherical in nature, and well segregated without any agglomeration and with an average size of $30 \mathrm{~nm}$. X-ray powder diffraction showed a polycrystalline nature and face centered cubic lattice and revealed characteristic diffraction peaks indicating the formation of CuONPs. Fourier-transform infrared spectra confirmed the presence of capping proteins on the NP surface that act as stabilizers. All CuONPs manifested antimicrobial activity against various types of Gram-negative; Gram-positive bacteria; and fungi pathogen microorganisms including Escherichia coli, Staphylococcus aureus, and Candida albicans. The cost-effective and eco-friendly biosynthesis of these CuONPs make them particularly attractive in several application from nanotechnology to biomedical science.
\end{abstract}

Keywords: green synthesis; biomaterials; metal; antibiotics; nanotechnology

\section{Introduction}

With the beginning of the 21st century, nanobiotechnology entered the scientific spotlight as a discipline for innovative materials and applications. Nanoparticles (hereafter called NPs) are becoming the fundamental building blocks of nanotechnology. Their small dimensions and high surface area to volume enable them to exhibit novel chemical and physical properties. Consequently, NPs can be used in applications that are different from those of their bulk materials, including but not limited to electrical resistivity and conductivity, chemical reactivity, and diverse and versatile biological processes [1,2]. Most research work in this field focused on silver and gold NPs. However, special interest has been taken in other metal NPs because these particles are being widely used as industrial catalysts, in chemical sensing devices, in medical applications, in cosmetics, and as microelectronics [1-4]. Among these, interest is growing for NPs from copper due to the attractive physical and chemical properties. Specifically, there are different types of copper NPs that can be synthesized via multiple methods: CuNPs, cupric oxide nanoparticles (CuONPs), and cuprous oxide nanoparticles 
$\left(\mathrm{Cu}_{2} \mathrm{ONPs}\right)$ [5-8]. In particular, $\mathrm{CuONPs}$ are p-type oxide semiconductors that, for its catalytic, optical, magnetic, and electrical properties, are largely used as catalysts; as sensors; in optoelectronics; as photocatalyst; and in antibacterial, antifungal, antiviral, anticancer, antioxidant, or drug delivery applications, among other $[9,10]$. In addition, CuONPs are significantly less toxic, with values L50 values reported between 64 and $840 \mathrm{mg} / \mathrm{L}$ vs. between 0.22 and 24 $\mathrm{mg} / \mathrm{L}$ for CuNPs [11]. For the synthesis of CuNPs, many methods have been employed such as thermal evaporation, chemical synthesis, electrochemical synthesis, solvothermal route, and vapor-liquid-solid growth [5-10]. However, physical methods have some disadvantages such as the requirement of expensive and complicated vacuum techniques. Chemical synthesis limits NP applications in clinical fields due to the usage of toxic chemicals that may lead to environmental and biological hazards. By contrast, CuNP green synthesis is eco-friendly and cost effective and does not use toxic chemicals, which make CuNPs attractive in biomedical applications.

$\mathrm{Cu}$ is an essential micronutrient for living cells because it is a constituent of many metalloenzymes including cytochrome-c oxidase and superoxide dismutase (SOD) [12]. However, $\mathrm{Cu}$ can also generate reactive oxygen species $[13,14]$, it can be poisonous at high concentrations, and can reduce microorganism growth. It is well established that, when microbes are kept in a toxic metal environment, they evolve mechanisms to survive in harsh conditions by transforming toxic metal ions into their corresponding nontoxic forms such as metal sulfide/oxides. For example, a new Alcanivorax sp. isolated from a shallow hydrothermal vent was resistant to copper toxicity [15]. A large group of biological resources such as bacteria, yeasts, fungi, algae, and plants can be used for the synthesis of NPs from metal ions; however, the detailed mechanisms involved in nanoscale transformation are not well established. Among all biological systems used until now, bacteria have acquired significant attention as they are easy to culture, are able to produce extracellular NPs with easy downstream processing (as purification steps), and have short generation times for NP synthesis. Furthermore, a large group of biological resources such as bacterial biomolecules acts as a reducing and stabilizing agent for NP synthesis, limiting particle growth, and prohibiting agglomeration, resulting in the formation of desired NPs. In this regard, copper oxide nanoparticles have different applications, such as antibacterial, antifungal, antiviral, anticancer, antioxidant, and drug delivery applications [10].

In the present study, we report the biosynthesis of CuONPs at low temperatures from bacterial strains isolated from a consortium associated with the Antarctic ciliate Euplotes focardii [16]. This microorganism is a free-swimming ciliate, endemic of the oligotrophic coastal sediments of the Terra Nova Bay [17]. All bacterial strains are identified as Marinomonas (MM) [18], Rhodococcus (RH), Pseudomonas (PM) [19-21], Brevundimonas (BM), and Bacillus (BC). All were named with the "ef1" suffix (ef stands for Euplotes focardii and 1 indicates that is the first strain of the genus isolated from this organism).

Although Antarctica is regarded as the last uncontaminated continent, it is not completely free from pollution [22]. Despite isolation of the continent by natural barriers such as circumpolar atmospheric and oceanic currents [23], contaminants such as heavy metals, pesticides, and other persistent organic pollutants (POPs) could reach Antarctica via long range atmospheric transport (LRAT) from other continents in the southern hemisphere and even beyond [24]. Previous work reported evidence that bacteria from Antarctica developed resistance to heavy metals $[25,26]$. Our results support this evidence since these bacterial strains shows resistance to up to $5 \mathrm{mM}$ of $\mathrm{CuSO}_{4}$ and produced $\mathrm{CuONPs}$. Our study highlights an efficient strategy in obtaining bionanomaterials that can be used as antibiotics against a large number of drug-resistant pathogens bacteria, which has created serious concern across the globe due to the limited choices in antibiotic treatment [27].

\section{Results and Discussion}

\subsection{Copper Tolerance and Growth Assessment for the Bacterial Strains}

As a first step in this work, we assessed the $\mathrm{Cu}$ tolerance of all bacterial strains under study. Tables 1 and 2 report the bacterial growth assessments at different $\mathrm{CuSO}_{4}$ concen- 
trations and the maximum tolerated concentrations (MTCs) of heavy metals, respectively. Our results indicate that all strains tolerate $\mathrm{CuSO}_{4}$ up to $3.5-4 \mathrm{mM}$.

Table 1. Growth assessment of bacteria with various concentrations of $\mathrm{CuSO}_{4}$. High growth: +++, medium growth: ++ , low growth: + , and no growth: - .

\begin{tabular}{ccccccccccccccc}
\hline \multicolumn{10}{c}{ CuSO $_{4}$ Concentration $(\mathrm{mM})$} \\
\hline Organisms & $\mathbf{0 . 0}$ & $\mathbf{0 . 5}$ & $\mathbf{1}$ & $\mathbf{1 . 5}$ & $\mathbf{2}$ & $\mathbf{2 . 5}$ & $\mathbf{3}$ & $\mathbf{3 . 5}$ & $\mathbf{4}$ & $\mathbf{4 . 5}$ & $\mathbf{5}$ & $\mathbf{5 . 5}$ & $\mathbf{6}$ \\
\hline Marinomonas ef1 & +++ & +++ & +++ & +++ & +++ & +++ & ++ & ++ & ++ & ++ & + & - & - \\
\hline Rhodococcus ef1 & +++ & +++ & +++ & +++ & +++ & +++ & +++ & ++ & + & + & - & - & - \\
\hline Pseudomonas ef1 & +++ & +++ & +++ & +++ & ++ & ++ & + & + & - & - & - & - & - \\
\hline Brevundimonas ef1 & +++ & +++ & +++ & +++ & +++ & ++ & ++ & ++ & ++ & + & + & - & - \\
\hline Bacillus ef1 & +++ & +++ & +++ & +++ & +++ & ++ & ++ & + & + & - & - & - & - \\
\hline
\end{tabular}

Table 2. Maximum tolerated $\mathrm{CuSO}_{4}$ concentrations (MTCs).

\begin{tabular}{cc}
\hline Organisms & $\mathbf{C u S O}_{\mathbf{4}}(\mathbf{m M})$ \\
\hline Marinomonas ef1 & 5 \\
\hline Rhodococcus ef1 & 4.5 \\
\hline Pseudomonas ef1 & 3.5 \\
\hline Brevundimonas ef1 & 5 \\
\hline Bacillus ef1 & 4 \\
\hline
\end{tabular}

We also monitored bacterial growth in the presence of increasing $\mathrm{CuSO}_{4}$ concentrations for each strain (Figure S1): increasing copper concentrations decreased the growth rate of all bacteria tested. The highest growth inhibition effect is visible at concentrations above $3.5 \mathrm{mM}$, in particular, for Pseudomonas ef1 and Bacillus ef1 (Figure S1).

\subsection{Biosynthesis of $\mathrm{CuNPS}$}

All of the tested bacteria showed resistance to $\mathrm{Cu}$ up to $3.5 \mathrm{mM}$; thus, we reasoned that these strains may be able to synthesize copper NPs. With the addition of $1 \mathrm{mM}$ $\mathrm{CuSO}_{4}$ (final concentration) in the reaction medium, we observed a gradual change in the solution color from cyan to brown over a $48 \mathrm{~h}$ period of time (Figure S2). A similar change in color has been reported after the addition of $5 \mathrm{mM} \mathrm{CuSO}_{4}$ to a flask containing Morganella sp. [28], or three different species of Penicillium and the white-rot fungus Stereum hirsutum [29]. Therefore, our result suggests the formation of copper NPs from $\mathrm{CuSO}_{4}$ through microbial metabolisms.

\subsection{Ultraviolet-Visible Absorption Spectroscopy (UV-Vis), Dynamic Light Scattering (DLS) Analysis, and Zeta Potential Measurements of CuNPs}

UV-vis spectral analysis is the most important analysis method to detect the surface plasmon resonance (SPR) property of biosynthesized CuNPs. We applied UV-vis spectroscopy to all of the samples obtained from the different strains: a sharp peak with maximum absorption in the range of 381-383 nm was recorded in each sample and can be attributed to the formation of CuNPs [30] (Figure S3).

Our results are in agreement with previous reports on bacterial synthesized CuNPs. Indeed, broad absorption spectra peaks were observed at around $365 \mathrm{~nm}$ for CuNPs synthesized from Escherichia coli [31], at $310 \mathrm{~nm}$ for Eichhornia crassipes [32], and at $360 \mathrm{~nm}$ for CuNPs synthesized from Ixora coccinea leaf extracts [30].

Dynamic light scattering (DLS) was also performed to determine the size distribution and zeta potential for biosynthesized CuNPs. All of the average diameters are reported in Figure 1 for all of the different bacterial strains. 

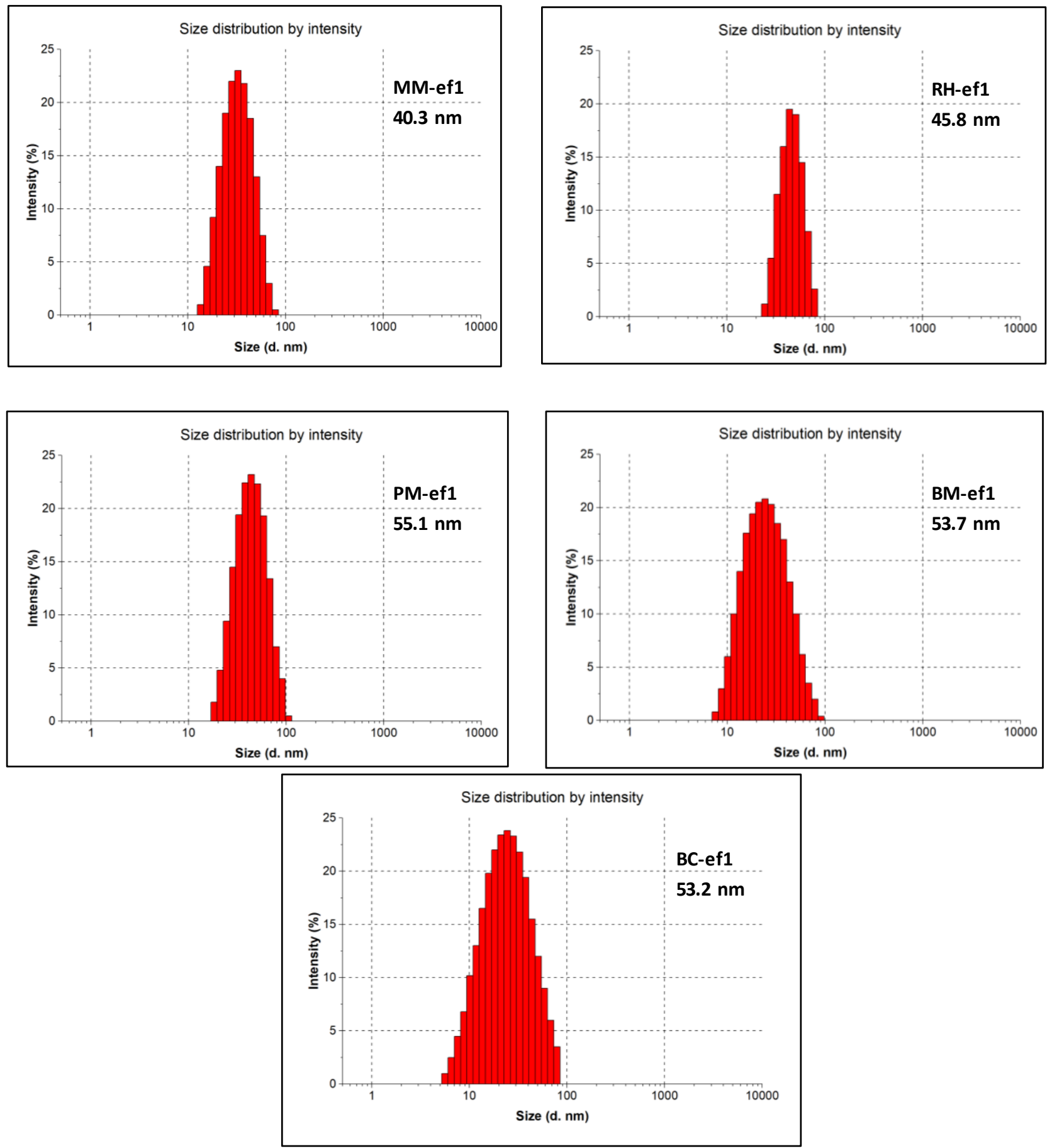

Figure 1. DLS of bio-CuNPs synthesized from the different bacterial strains: Marinomonas (MM), Rhodococcus (RH), Pseudomonas (PM), Brevundimonas (BM), and Bacillus (BC).

Similarly, the zeta potential values of CuNPs are in the range from $-23.2 \mathrm{mV}$ to $-33.8 \mathrm{mV}$ (Table 3). The zeta potential data demonstrated that biosynthesized NPs were stable in a liquid medium, and therefore, the tested bacterial strains represent a good source for production of narrow-sized CuNPs. The stability may be due to NP capping by biomolecules produced as byproducts. The higher negative zeta potential values obtained, thus, confirmed the repulsion between particles to thwart agglomeration. 
Table 3. Zeta potential of CuNPs obtained from different strains.

\begin{tabular}{cc}
\hline Organisms & Zeta Potential (mV) \\
\hline Marinomonas ef1 & -23.2 \\
\hline Rhodococcus ef1 & -33.8 \\
\hline Pseudomonas ef1 & -33.1 \\
\hline Brevundimonas ef1 & -33.6 \\
\hline Bacillus ef1 & -25.1 \\
\hline
\end{tabular}

\subsection{XRD and FTIR Analyses}

The powered XRD analysis of synthesized CuNPs with different strains was applied in order to investigate the crystalline phase of $\mathrm{Cu}$ nanostructures. In Figure 2, all XRD profiles of CuNPs are reported in comparison with simulation data (black line defined as theoretical). The obtained spectra reveal characteristic diffraction peaks indicating the formation of $\mathrm{CuO}$ in monoclinic and crystalline phase (the specific explanations are reported in Appendix A). In the $\mathrm{CuO}$ monoclinic structure, each $\mathrm{Cu}$ atom is situated at the center of four oxygen atoms positioned at the vertices of a rectangle with oxygen atoms at the center of a tetrahedron of $\mathrm{Cu}$ atoms [31]. The slight differences in peak positions with respect to the simulation data indicate that the different coatings of the obtained nanoparticles promote defects in the crystals, giving different crystalline orientations with respect to the theoretical ones. In addition, the XRD pattern demonstrate the absence of impurity and of peaks related to $\mathrm{Cu}(\mathrm{OH})_{2}$ or $\mathrm{Cu}_{2} \mathrm{O}$ phases (see Appendix A for more details).

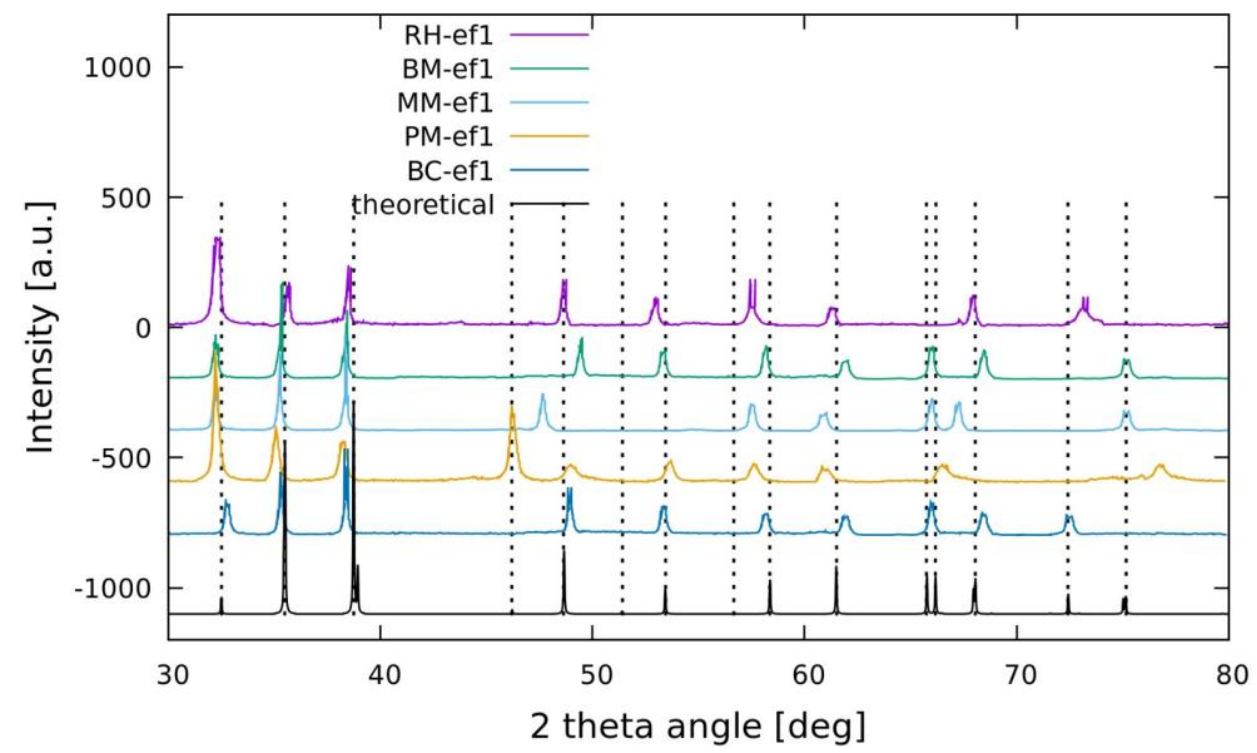

Figure 2. XRD profile of biosynthesized CuONPs. The obtained spectra reveal characteristic diffraction peaks indicating the formation of $\mathrm{CuO}$ in all bacterial NPs in comparison with simulation peaks (black line defined as the theoretical peaks).

FTIR spectroscopy was carried out to verify the possible involvement of functional groups or biomolecules in $\mathrm{CuONP}$ formation and stabilization. This technique is a powerful tool used to identify the chemical bonds in a molecule by producing an IR spectrum that is similar to a molecular fingerprint. The FTIR spectra of CuONPs biosynthesized by MM-ef1, RH-ef1, PS-ef1, BM-ef1, and BC-ef1 were obtained in the range between 400 and $4000 \mathrm{~cm}^{-1}$ (Figures S4-S8).

The IR spectra suggested that the biomolecules interacted with the biosynthesized $\mathrm{CuONPs}$. The distinct bands analyzed indicated the presence of $-\mathrm{OH},-\mathrm{NH}$, and $-\mathrm{CH}_{2}$ scissor vibrations of aliphatic compounds and $\mathrm{C}=\mathrm{C}$ bonds inside the biomolecules. From 
the analysis of the peaks, a carbonyl group $(\mathrm{C}=\mathrm{O})$ of the amide functional group was also detected. Therefore, the presence of carbonyl and $\mathrm{NH}$ groups is important for stabilization of the nanoparticles. In fact, the doublet of electrons present on both groups can be useful for the electrostatic stabilization of CuONP nanoparticles and thus to function as a capping agent [32].

\subsection{Transmission Electron Microscopy (TEM)}

Controlling NP size distribution is important for many applications, in particular for antimicrobial activity. We applied TEM analysis (Figure 3) to obtain additional insights in the morphology and size of the CuONPs from all of the strains.

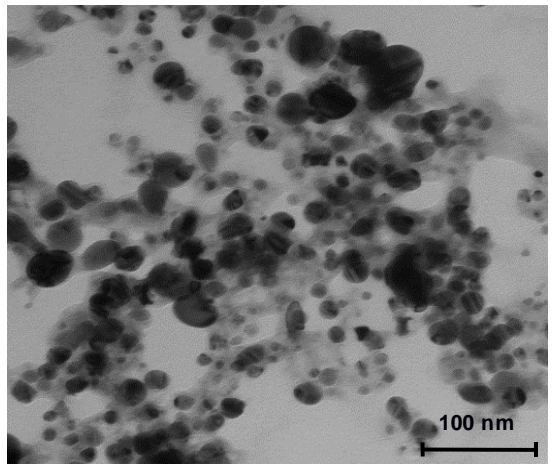

Marinomonas ef 1

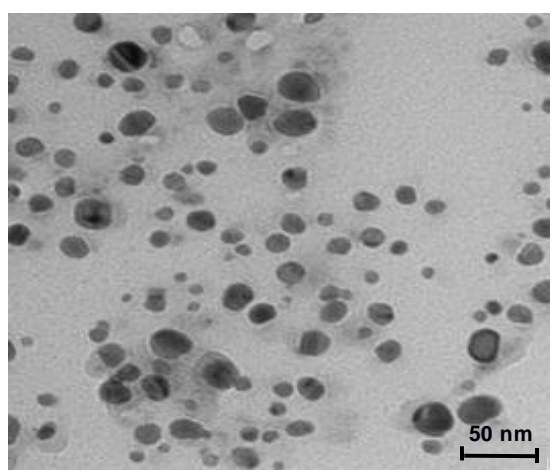

Pseudomonas ef1

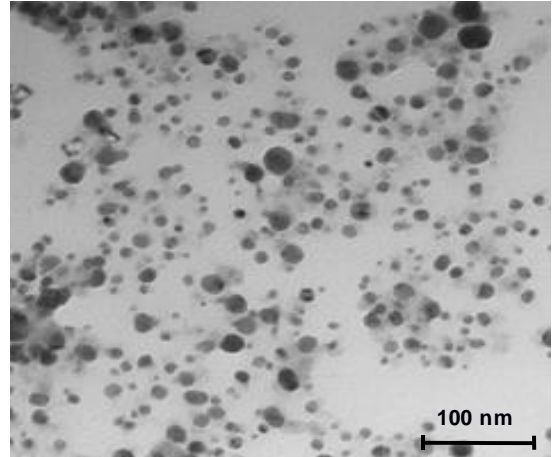

Rhodococcus ef1

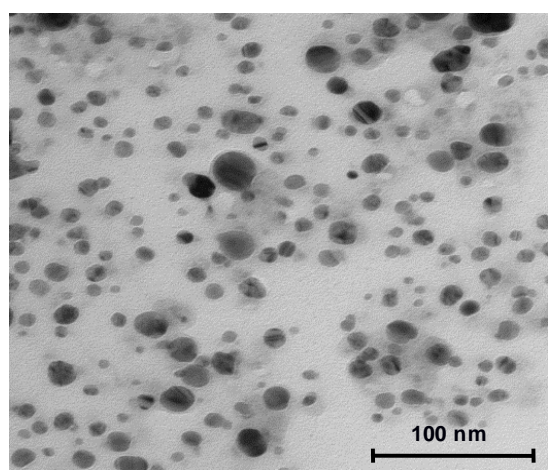

Brevundimonas ef1

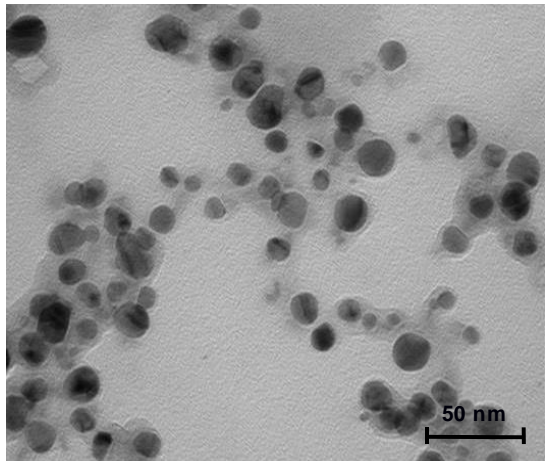

Bacillus ef1

Figure 3. TEM images of biosynthesized CuONPs from the five bacterial strains: Marinomonas ef1, Rhodococcus ef1, Pseudomonas ef1, Brevundimonas ef1, and Bacillus ef1.

TEM micrographs of all bacterial CuONPs showed that the particles are monodispersed, spherical/ovoidal shapes. The micrographs suggested particle sizes around $10 \mathrm{~nm}$ to $70 \mathrm{~nm}$, with the average size being $40 \mathrm{~nm}$. The irregular shape and change in dimensions observed using different bacteria during the synthesis depends by the differ- 
ent bacterial metabolisms and of the secondary compounds produced that surround the nanoparticles. These compounds act as capping and dispersing agents influencing the shape and dimension of the nanostructures. All of the nanoparticles are well segregated except for the nanoparticles produced by Marinomonas ef1, where larger aggregates are observed (Figure 3).

\subsection{Antimicrobial Activity of the Bacterial CuONPs}

It has been reported that CuNPs of about $30 \mathrm{~nm}$ are more active against pathogens than those of smaller size [33]. CuONPs have received much attention in a wide range of applications, including their use as antimicrobial agents [9,34-37].

To test the potential antimicrobial activity of our biosynthesized CuONPs, we performed the disk diffusion test against Staphylococcus aureus, Escherichia coli (EC), Klebsiella pneumoniae (KP), Pseudomonas aeruginosa (PA), Proteus mirabilis (PrM), Citrobacter koseri (CK), Acinetobacter baumanii (AB), Serratia marcescens (SM), Candida albicans (CA), and Candida parapsilosis (CP).

The results of the disk diffusion test are reported in Figure 4, in which the presence of a clear zones around the CuONP disk is clearly visible, suggesting that all nanoparticles were able to inhibit the growth of bacterial and fungal pathogens.

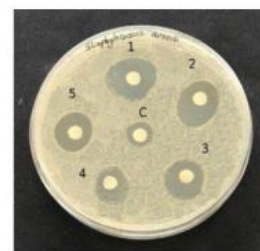

SA

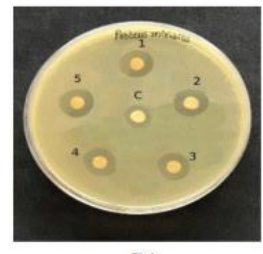

PA

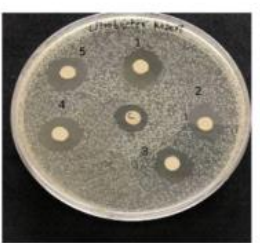

CK

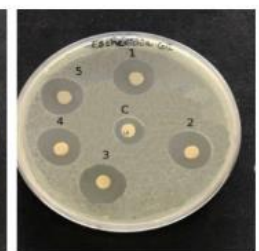

EC

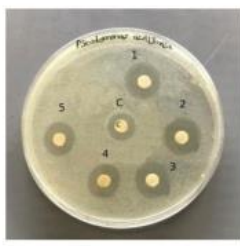

PrM

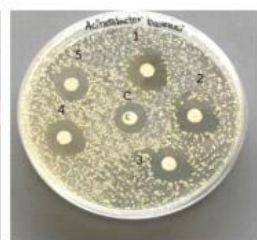

$\mathbf{A B}$

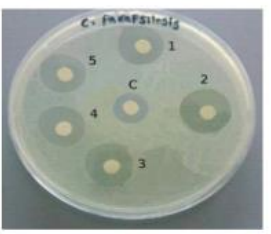

CP

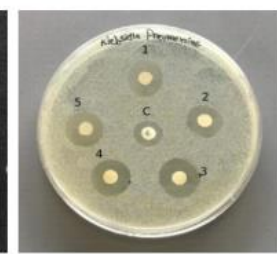

KP

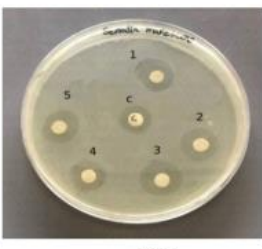

SM

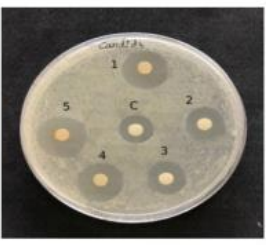

CA

Figure 4. Antibacterial activity of bio-CuONPs against pathogenic bacteria: Staphylococcus aureus (SA), Escherichia coli (EC), Klebsiella pneumoniae (KP), Pseudomonas aeruginosa (PA), Proteus mirabilis (PrM), Serratia marcescens (SM), Citrobacter koseri (CK), Acinetobacter baumanii (AB), Candida albicans (CA), and Candida parapsilosis (CP). Kirby-Bauer disk diffusion test. 1. MM-ef1, 2. RH-ef1, 3. BM-ef1, 4. PM-ef1, 5. BC-ef1, and C-control (1 $\mathrm{mM}$ of $\left.\mathrm{CuSO}_{4}\right)$.

All of the results for inhibition zone variation are summarized in Table 4 as a comparison with negative controls. 
Table 4. Inhibition zone variation due to antibacterial activity of bio-CuONPs.

\begin{tabular}{|c|c|c|c|c|c|c|}
\hline & MM-ef1 & RH-ef1 & BM-ef1 & PM-ef1 & BC-ef1 & $\mathrm{CuSO}_{4}$ \\
\hline & \multicolumn{6}{|c|}{ Gram-Positive Bacteria } \\
\hline \multirow[t]{2}{*}{$S A$} & $16 \pm 0.2 *$ & $16 \pm 0.3^{*}$ & $16 \pm 0.1^{*}$ & $13 \pm 0.4^{*}$ & $15 \pm 0.2 *$ & $9 \pm 0.3^{\circ}$ \\
\hline & \multicolumn{6}{|c|}{ Gram-Negative Bacteria } \\
\hline$E C$ & $15 \pm 0.2 *$ & $16 \pm 0.4^{*}$ & $17 \pm 0.2 *$ & $16 \pm 0.3^{*}$ & $16 \pm 0.2 *$ & $10 \pm 0.1^{\circ}$ \\
\hline$K P$ & $16 \pm 0.1 *$ & $15 \pm 0.1$ * & $17 \pm 0.3 *$ & $16 \pm 0.4^{*}$ & $16 \pm 0.3 *$ & $10 \pm 0.3^{\circ}$ \\
\hline$P M$ & $16 \pm 0.3^{*}$ & $17 \pm 0.4^{*}$ & $16 \pm 0.4^{*}$ & $15 \pm 0.4^{*}$ & $15 \pm 0.2 *$ & $11 \pm 0.2^{\circ}$ \\
\hline $\operatorname{PrM}$ & $15 \pm 0.1 *$ & $15 \pm 0.2 *$ & $14 \pm 0.2 *$ & $15 \pm 0.2 *$ & $15 \pm 0.4$ * & $10 \pm 0.2^{\circ}$ \\
\hline CK & $16 \pm 0.3^{*}$ & $15 \pm 0.3^{*}$ & $15 \pm 0.2 *$ & $16 \pm 0.2 *$ & $16 \pm 0.3 *$ & $10 \pm 0.1^{\circ}$ \\
\hline$A B$ & $16 \pm 0.2 *$ & $17 \pm 0.4^{*}$ & $15 \pm 0.1 *$ & $16 \pm 0.2 *$ & $15 \pm 0.2 *$ & $11 \pm 0.2^{\circ}$ \\
\hline \multirow[t]{2}{*}{$S M$} & $15 \pm 0.2 *$ & $15 \pm 0.2 *$ & $15 \pm 0.2 *$ & $14 \pm 01 *$ & $14 \pm 0.2 *$ & $10 \pm 0.3^{\circ}$ \\
\hline & \multicolumn{6}{|c|}{ Fungi } \\
\hline$C A$ & $18 \pm 0.4^{*}$ & $16 \pm 0.4^{*}$ & $16 \pm 0.4^{*}$ & $17 \pm 0.4^{*}$ & $18 \pm 0.4$ * & $11 \pm 0.2^{\circ}$ \\
\hline$C P$ & $17 \pm 0.4$ * & $19 \pm 0.2 *$ & $17 \pm 0.4^{*}$ & $16 \pm 0.4^{*}$ & $16 \pm 0.4$ * & $11 \pm 0.2^{\circ}$ \\
\hline
\end{tabular}

Inhibition zone (Ø, in $\mathrm{mm}$ ) with ${ }^{*} \mathrm{CuONPs}$ and with ${ }^{\circ} \mathrm{CuSO}_{4}$ (negative control).

We further evaluated the CuONP antibacterial activity by estimating the minimum inhibitory concentration (MIC) and minimum bactericidal concentration (MBC) values using the plate microdilution method [38]. The MIC is defined as the lowest concentration of antibacterial agent needed that inhibits the growth of bacteria. As showed in Table 5 and Figures S9-S13, the CuONPs synthesized by all bacteria showed an MIC against the Gram-negative bacteria from 3.12 to $25 \mu \mathrm{g} / \mathrm{mL}$, against Gram-positive bacteria from 12.5 to $25 \mu \mathrm{g} / \mathrm{mL}$, and against fungi from 12.5 to $25 \mu \mathrm{g} / \mathrm{mL}$. MBC is defined as the lowest concentration of an antibacterial agent that kills the bacteria (no growth was observed on the agar plate). In the study, MBC values ranged from 12.5 to $25 \mu \mathrm{g} / \mathrm{mL}$. These analyses showed that these $\mathrm{CuONPs}$ possess strong antimicrobial activity. The different MIC and $M B C$ values could be attributed to the different shapes and dimensions of the nanoparticles determining their antibacterial activity.

Table 5. MIC and MBC values ( $\mu \mathrm{g} / \mathrm{mL}$ ) of the CuONPs synthesized by MM-ef1, RH-ef1, PM-228 ef1, BM-ef1, and BC-ef1 against pathogenic bacteria.

\begin{tabular}{|c|c|c|c|c|c|c|c|c|c|c|}
\hline & \multicolumn{2}{|c|}{ MM-ef1 } & \multicolumn{2}{|c|}{ RH-ef1 } & \multicolumn{2}{|c|}{ BM-ef1 } & \multicolumn{2}{|c|}{ PM-ef1 } & \multicolumn{2}{|c|}{ BC-ef1 } \\
\hline & MIC & MBC & MIC & MBC & MIC & MBC & MIC & MBC & MIC & MBC \\
\hline & \multicolumn{10}{|c|}{ Gram Positive Bacteria } \\
\hline \multirow[t]{2}{*}{$S A$} & $2.5 \pm 0.2$ & $25 \pm 0.4$ & $12.5 \pm 0.2$ & $25 \pm 0.2$ & $25 \pm 0.2$ & $25 \pm 0.4$ & $12.5 \pm 0.4$ & $25 \pm 0.2$ & $12.5 \pm 0.4$ & $12.5 \pm 0.1$ \\
\hline & \multicolumn{10}{|c|}{ Gram Negative Bacteria } \\
\hline$E C$ & $12.5 \pm 0.2$ & $25 \pm 0.3$ & $12.5 \pm 0.4$ & $25 \pm 0.3$ & $25 \pm 0.4$ & $25 \pm 0.3$ & $25 \pm 0.3$ & $25 \pm 0.2$ & $12.5 \pm 0.2$ & $12.5 \pm 0.2$ \\
\hline$K P$ & $12.5 \pm 0.1$ & $25 \pm 0.5$ & $6.25 \pm 0.3$ & $12.5 \pm 0.2$ & $12.5 \pm 0.2$ & $25 \pm 0.4$ & $12.5 \pm 0.2$ & $25 \pm 0.4$ & $6.25 \pm 0.2$ & $6.25 \pm 0.2$ \\
\hline$P M-s p$ & $6.25 \pm 0.2$ & $12.5 \pm 0.2$ & $12.5 \pm 0.2$ & $25 \pm 0.4$ & $12.5 \pm 0.2$ & $25 \pm 0.3$ & $12.5 \pm 0.1$ & $12.5 \pm 0.4$ & $12.5 \pm 0.3$ & $12.5 \pm 0.4$ \\
\hline$P M$ & $3.12 \pm 0.1$ & $6.25 \pm 0.2$ & $6.25 \pm 0.2$ & $12.5 \pm 0.1$ & $6.25 \pm 0.1$ & $12.5 \pm 0.2$ & $6.25 \pm 0.2$ & $12.5 \pm 0.2$ & $6.25 \pm 0.1$ & $12.5 \pm 0.1$ \\
\hline$C K$ & $6.25 \pm 0.2$ & $6.25 \pm 0.1$ & $12.5 \pm 0.3$ & $12.5 \pm 0.2$ & $12.5 \pm 0.2$ & $25 \pm 0.4$ & $6.25 \pm 0.1$ & $12.5 \pm 0.4$ & $12.5 \pm 0.4$ & $12.5 \pm 0.1$ \\
\hline$A B$ & $12.5 \pm 0.2$ & $12.5 \pm 0.2$ & $12.5 \pm 0.2$ & $12.5 \pm 0.2$ & $12.5 \pm 0.2$ & $25 \pm 0.4$ & $12.5 \pm 0.2$ & $12.5 \pm 0.2$ & $12.5 \pm 0.2$ & $12.5 \pm 0.4$ \\
\hline \multirow[t]{2}{*}{$S M$} & $6.25 \pm 0.1$ & $12.5 \pm 0.4$ & $3.12 \pm 0.1$ & $12.5 \pm 0.2$ & $6.25 \pm 0.1$ & $12.5 \pm 0.2$ & $6.25 \pm 0.1$ & $12.5 \pm 0.2$ & $6.25 \pm 0.2$ & $12.5 \pm 0.2$ \\
\hline & \multicolumn{10}{|c|}{ Fungi } \\
\hline$C A$ & $25 \pm 0.4$ & $25 \pm 0.5$ & $12.5 \pm 0.2$ & $25 \pm 0.4$ & $12.5 \pm 0.2$ & $25 \pm 0.4$ & $25 \pm 0.2$ & $25 \pm 0.4$ & $12.5 \pm 0.2$ & $25 \pm 0.3$ \\
\hline$C P$ & $12.5 \pm 0.4$ & $25 \pm 0.3$ & $25 \pm 0.2$ & $25 \pm 0.2$ & $12.5 \pm 0.1$ & $25 \pm 0.2$ & $12.5 \pm 0.3$ & $25 \pm 0.1$ & $6.25 \pm 0.2$ & $12.5 \pm 0.2$ \\
\hline
\end{tabular}


It was observed that the bactericidal property of CuONPs is due to the generation of a large number of small molecules containing reactive oxygen species (ROS) by the nanoparticles attached to the bacterial cells. Although cells can detoxify ROS species within certain limits by promoting antioxidant activity such as specific enzymes or small molecules (e.g., ascorbic acid), the formation and accumulation of ROS species largely increased when the cell was constitutively exposed to intracellular oxidative stress [36].

\section{Materials and Methods}

\subsection{Culture and Chemicals}

All bacterial strains used in this work were isolated from a consortium associated with the Antarctic ciliate E. focardii and identified as Marinomonas, Rhodococcus, Pseudomonas, Brevundimonas, and Bacillus $[17,18,20]$. All strains were grown at $22^{\circ} \mathrm{C}$ on agarized or liquid Luria-Bertani (LB) medium (tryptone $10 \mathrm{~g} / \mathrm{L}$, yeast extract $5 \mathrm{~g} / \mathrm{L}$, and $\mathrm{NaCl} 5 \mathrm{~g} / \mathrm{L}$ ). All media were purchased from Liofilchem. Analytical grade copper (II) sulfate pentahydrate salt, $\mathrm{CuSO}_{4} \cdot 5 \mathrm{H}_{2} \mathrm{O}$, and the other chemicals were purchased from Sigma Aldrich (Sigma Aldrich, St. Louis, MO, USA).

\subsection{Determination of Copper Maximum Tolerated Concentrations (MTCs)}

Copper $(\mathrm{Cu})$ maximum tolerated concentrations (MTCs) were determined for each isolate on agarized LB medium in the presence of increasing concentrations (from 0 to $6 \mathrm{mM}$ ) of $\mathrm{CuSO}_{4}$. With a sterile inoculating loop, each bacterial isolate was streaked on the $\mathrm{Cu}$ incorporated agarized LB medium. The plates were incubated at $22{ }^{\circ} \mathrm{C}$ and inspected at intervals up to $72 \mathrm{~h}$. The MTCs were noted when the isolate failed to show growth on the plates after three days of incubation. All experimental setups were prepared in triplicate.

\subsection{Estimation of Bacterial Growth Inhibition by Copper}

Bacterial growth $\mathrm{Cu}$ inhibition was determined by monitoring the optical density at regular time intervals at $600 \mathrm{~nm}$ using a Shimadzu UV 1800 spectrophotometer. In total, $0.5 \mathrm{~mL}$ of an overnight active culture adjusted to OD600 $=0.1$ was incorporated into $50 \mathrm{~mL}$ of $\mathrm{LB}$ medium supplemented with increasing concentrations of $\mathrm{CuSO}_{4}$ from 0 up to the maximum concentration tolerated by each bacterium. Inoculated flasks were incubated on a rotary shaker at $22{ }^{\circ} \mathrm{C}$. All experimental setups were prepared in triplicate.

\subsection{Biosynthesis of CuNPs}

Each strain was inoculated in LB medium $(100 \mathrm{~mL})$ and incubated at $22{ }^{\circ} \mathrm{C}$ on a rotatory shaker (200 rpm). After $24 \mathrm{~h}, \mathrm{CuSO}_{4} \cdot 5 \mathrm{H}_{2} \mathrm{O}$ was added to the microbial cell culture to a final concentration of $1 \mathrm{mM}$. The reaction mixture was incubated for 24 to $48 \mathrm{~h}$ on a rotatory shaker at $150 \mathrm{rpm}$ at $22{ }^{\circ} \mathrm{C}$. $\mathrm{LB}$ medium with $1 \mathrm{mM} \mathrm{CuSO}_{4}$ without the organism or heat-killed bacterial cultures were maintained as a control. During the incubation period, the solution color change from blue to dark green (which is indicative of the reduction of $\mathrm{CuSO}_{4}$ to CuNPs) was monitored. The formation of CuNPs was also recorded by absorption spectra in the wavelength range of $200-800 \mathrm{~nm}$ at room temperature $\left(23^{\circ} \mathrm{C}\right)$ using a Shimadzu UV 1800 spectrophotometer.

\subsection{Purification of $\mathrm{CuNPs}$}

After incubation, the culture was centrifuged at $5000 \mathrm{rpm}$ at $4{ }^{\circ} \mathrm{C}$ for $20 \mathrm{~min}$ in a Beckman J2-21 with swinging rotor to separate the cell pellet from the cell-free supernatant. Nanoparticles were purified from both the supernatant and pellet. To recover CuNPs present in the cell-free supernatant, the solution was centrifuged at $17,000 \times g$ for $15 \mathrm{~min}$ in the same centrifuge with a fixed rotor. The CuNPs containing the pellet was then resuspended in double-distilled water $\left(\mathrm{ddH}_{2} \mathrm{O}\right)$ and washed twice by repeated centrifugation steps.

To recover CuNPs from the cell pellet, ultrasonic wave shocks of short durations (15 s) were given to the $\mathrm{ddH}_{2} \mathrm{O}$-suspended pellet to rupture the microbial cell wall. After 
sonication, the sample was centrifuged $5000 \mathrm{rpm}$ for $20 \mathrm{~min}$ (Beckman J2-21, Fullerton, California) and the NPs were recovered from the supernatant. This step was repeated three times to completely remove the cell debris from the supernatant. To recover CuNPs present in the cell-free supernatant, the solution was centrifuged at $17,000 \times g$ for $15 \mathrm{~min}$ in the same centrifuge with a fixed rotor. After being washed twice with deionized water and dried at $80{ }^{\circ} \mathrm{C}$ in an oven, the CuNPs were used for further characterization and experiments.

3.6. Dynamic Light Scattering, Zeta Potential Measurement, Transmission Electron Microscopy (TEM), X-ray Diffraction Analysis (XRD), and Fourier-Transform Infrared Spectroscopy (FTIR) Analyses

Zetasizer Nano ZS, (Malvern Instruments Ltd., Malvern, UK) was used to determine the size distribution of particles by measuring dynamic fluctuations of light scattering intensity caused by the Brownian motion of the particles. All measurements were carried out in triplicate with a temperature equilibration time of $2 \mathrm{~min}$ at $25^{\circ} \mathrm{C}$. Additionally, NP surface charge was measured using the zeta potential. The morphologies of the biosynthesized CuNPs were observed on a JEOL transmission electron microscope (TEM) system operating at $200 \mathrm{kV}$ (JEM-2100, Hitachi Limited, Tokyo, Japan), with the acquirement of the particle size distribution ascertained from TEM micrographs based on professional software (Nano Measurer 1.2.5). The crystal structure of biosynthesized CuNPs was analyzed by powder X-ray diffraction (XRD) measurements performed using a Rigaku-D/MAX-PC 2500 X-ray diffractometer (Wilmington, MA, USA) with a $\mathrm{Cu} \mathrm{K} \alpha(\lambda=1.5405 \AA)$ in the $2 \theta$ range from 20 to $80^{\circ}$ at a scan rate of $0.03^{\circ} \mathrm{S}-1$. FTIR spectra (Kyoto, Japan) were recorded (Shimadzu IR Affinity-1) to identify the possible interactions between CuNPs and the biomolecule. Analysis was carried out in the range of $400-4000 \mathrm{~cm}^{-1}$ at the resolution of $4 \mathrm{~cm}^{-1}$.

\subsection{Kirby-Bauer Disk Diffusion Susceptibility Test, Minimum Inhibitory Concentration (MIC), and Minimum Bactericidal Concentration (MBC) Evaluation}

The antibacterial activity of biosynthesized CuNPs was tested against Staphylococcus aureus, Escherichia coli, Klebsiella pneumoniae, Pseudomonas aeruginosa, Proteus mirabilis, Citrobacter koseri, Acinetobacter baumanii, Serratia marcescens, Candida albicans, and Candida parapsilosis. All the strains were cultured in Mueller Hinton broth (MHB) (Merck, Darmstadt, Germany) at $37^{\circ} \mathrm{C}$. The antibacterial activity of CuNPs against the selected bacterial strains was assessed using the Kirby-Bauer disk diffusion susceptibility test method. Using a sterile cotton swab, the bacteria strains were spread on the Mueller-Hinton agar (MHA). The disks were loaded with $25 \mu \mathrm{L}(25 \mu \mathrm{g})$ of $1 \mathrm{mg} / \mathrm{mL}$ CuNP solution and $\mathrm{CuSO}_{4}$ solution $(1 \mathrm{mM})$ and dried. The disks were then placed on the agar plate and incubated at $37^{\circ} \mathrm{C}$. The inhibition zone was observed after $24 \mathrm{~h}$ of incubation.

The MIC and MBC estimations of the CuNPs were performed using the method described in the guideline of CLSI 2012 [38]. The MIC test was performed on a 96-well round bottom microtiter plate using standard broth microdilution methods, while the MBC test was performed on MHA plates. The bacterial inoculums were adjusted to the concentration of $0.5 \mathrm{McF}$ Farland units. For the MIC test, CuNP stock solution was prepared by ultrasonication in sterilized deionized water to reach $200 \mu \mathrm{g} / \mathrm{mL}$. A volume of $100 \mu \mathrm{L}$ of stock solution was serially diluted twofold in $100 \mu \mathrm{L}$ of MHB in the first row, and finally $100 \mu \mathrm{L}$ was discarded such that the first well in the row of the microtiter plate contained the highest concentration of CuNPs while the last well of the row contained the lowest concentration. Similarly, CuNPs were prepared in all of the rows. The positive control contained the medium and bacterial inoculums $\left(\mathrm{K}^{+}\right)$, and the negative control contained only the medium $\left(\mathrm{K}^{-}\right)$. The microtiter plate was then incubated at $37^{\circ} \mathrm{C}$ for $24 \mathrm{~h}$. The MIC value was defined as the lowest concentration of antibacterial agents that inhibits the growth of bacteria. The MBC was taken as the lowest concentration of antibacterial agents that completely kills the bacteria. To check $\mathrm{MBC}$, the suspension from each well of the microtiter plates was plated onto the MHA plate and were incubated at $37^{\circ} \mathrm{C}$ for $24 \mathrm{~h}$. The MBC value was taken as the lowest concentration with no visible growth on the MHA plate. 


\section{Conclusions}

In this study, we reported the production of monodisperse, small, and highly pure bio-CuONPs using the green reduction of $\mathrm{CuSO}_{4}$ at low temperatures, such as $22^{\circ} \mathrm{C}$, by using five Antarctic bacterial strains. The ability of these bacteria to synthesize CuONPs may represent a defense mechanism against this heavy metal. The results support the evidence that these bacterial strains are resistant to up to $5 \mathrm{mM}$ of $\mathrm{CuSO}_{4}$. All of the nanoparticles were fully characterized and tested for their antibacterial activity.

This study confirms that the tested Antarctic bacterial strains can be exploited in bioremediation to remove copper contamination from the environment and in the production of antibiotics against various types of pathogenic Gram-negative and Gram-positive bacteria, and fungi including Escherichia coli, Staphylococcus aureus, and Candida albicans. These results showed that the cost-effective and eco-friendly biosynthesis of these CuONPs make them particularly attractive in several applications including biomedical science.

\section{Patents}

The results of this paper are related to patent numbers 102019000014121 and 10201900 0024493. Marinomonas sp. ef1 and Rhodococcus sp. ef1 have been deposited at the Istituto Zooprofilattico Sperimentale della Lombardia e dell'Emilia Romagna "Bruno Ubertini" IZSLER according to the Budapest treaty under access No. DPS RE RSCIC 17 and DPS RE RSCIC 4. Breoundimonas sp ef1 and Bacilus sp ef1 have be deposited under access No. DPS RE RSCIC 23 and DPS RE RSCIC 24.

Supplementary Materials: The following are available online at https:/ /www.mdpi.com/article/10 $.3390 / \mathrm{md} 19050263 / \mathrm{s} 1$. Figure S1, Bacterial growth in the presence of increasing $\mathrm{CuSO}_{4}$ concentrations from 0.5 to $4 \mathrm{mM}$. Figure S2, Biosynthesis of Cu NPs from Marinomonas ef1, Rhododcoccus ef1, Pseudomonas ef1, Brevundimonas ef1, and Bacillus ef1. A. Control with a heat-killed bacterial culture using $1 \mathrm{mM}$ of $\mathrm{CuSO}_{4}$, B. control with the $\mathrm{LB}$ medium using $1 \mathrm{mM}$ of $\mathrm{CuSO}_{4}$, and C. biosynthesized CuNPs. Figure S3, UV - vis absorbance spectra of bio-CuONPs synthesized from (A) Rhodococcus ef1, (B) Pseudomonas ef1, (C) Brevundimonas ef1, (D) Bacillus ef1, and (E) Marinomonas ef1. Figures S4, FTIR spectrum of biosynthesized $\mathrm{CuO}$ NPs from Marinomonas ef1 and IR assignments. Figures S5, FTIR spectrum of biosynthesized CuO NPs from Rhodococcus ef1 and IR assignments. Figures S6, FTIR spectrum of biosynthesized CuO NPs from Pseudomonas ef1 and IR assignments. Figures S7, FTIR spectrum of biosynthesized CuO NPs from Brevundimonas ef1 and IR assignments. Figures S8, FTIR spectrum of biosynthesized CuO NPs from Bacillus ef1 and IR assignments. Figure S9, MIC values of Marinomonas CuO NPs. Figure S10, MIC values of Rhodococcus ef1 CuO NPs. Figure S11, MIC values of Brevundimonas ef1 CuO NPs. Figure S12, MIC values of Pseudomonas ef1 CuO NPs. Figure S13, MIC values of Bacillus ef $1 \mathrm{CuO}$ NPs.

Author Contributions: Study conception and design: M.S.J., J.A.N., S.P.; Acquisition of data: M.S.J. and J.A.N.; Analysis and interpretation of data: K.P.R., M.Z., R.G., A.M. and S.P.; Drafting of manuscript: M.S.J., J.A.N.; Critical revision: C.M., S.P. All authors have read and agreed to the published version of the manuscript.

Funding: This research work was supported by the European Commission Marie Sklodowska-Curie Actions H2020 RISE Metable-645693.

Acknowledgments: We are grateful to Ilidio Correia from the Faculty of Health Sciences, Universidade da Beira Interior, Covilhã (Portugal).

Conflicts of Interest: The authors declare no conflict of interest.

\section{Appendix A. Comments on the X-ray Diffraction (XRD) Structural Analysis}

The XRD analysis of Marinomonas ef1 synthesized CuO NPs show characteristic diffraction peaks at $2 \theta$ of $32.32^{\circ}, 35.18^{\circ}, 38.45^{\circ}, 48.10^{\circ}, 58.05^{\circ}, 61.21^{\circ}, 66.11^{\circ}, 67.56^{\circ}$ and $75.17^{\circ}$ which were assigned to (110), (111), (111), (202), (202), (113), (022), (220) and (004) planes respectively. All the peaks correspond to the face centered cubic (fcc) structure and were in 
consistence with the standard JCPDS (No.04-0836) data. The strong intensity diffraction peaks clearly indicate that the $\mathrm{CuO}$ NPs are highly crystalline and in monoclinic phase.

The XRD pattern of CuO NPs synthesized from Rhodococcus ef1 demonstrate that the $\mathrm{CuO}$ NPs is crystalline in nature. Also the spectrum is similar to that of pure $\mathrm{CuO}$, indicating the formation of single-phase $\mathrm{CuO}$ with monoclinic structure (JCPDS. 05-0661). In the present work, the diffraction patterns were observed to be at $2 \theta$ value of $32.37^{\circ}$, $35.39^{\circ}, 38.66^{\circ}, 48.61^{\circ}, 53.30^{\circ}, 58.21^{\circ}$ and $61.35^{\circ}$ and they were assigned to the corresponding (110), (111), (111), (202), (020), (202), (113), (113) and (113) planes, respectively. The reflection lines indicate the formation of monoclinic CuO NPs.

According to JCPDS data (80-0076), Pseudomonas ef1 exhibited diffraction peaks at $2 \theta$ of $32.49^{\circ}, 35.50^{\circ}, 38.70^{\circ}, 48.72^{\circ}, 58.28^{\circ}, 61.54^{\circ}, 66.24^{\circ}, 68.03^{\circ}$ and $75.16^{\circ}$ which were assigned to (110), (111), (111), (202), (202), (113), (311), (220) and (004) planes respectively corresponds to different planes of monoclinic phase of CuONPs and correspond to the face centered cubic (FCC) structure. Diffraction peaks clearly indicate that the CuONPs are highly crystalline.

The phase purity and structural characteristics of the synthesized $\mathrm{CuO}$ NPs from Brevundimonas ef1 showed the XRD Diffraction patterns appeared at $2 \theta$ of $32.42^{\circ}, 35.47^{\circ}$, $38.59^{\circ}, 48.66^{\circ}, 53.54^{\circ}, 58.69^{\circ}, 61.70^{\circ}, 66.01^{\circ}, 68.18^{\circ}$ and $75.19^{\circ}$ values and they were assigned to the corresponding (110), (002), (111), (112), (020), (202), (113), (310), (220) and (004) planes, respectively. The obtained XRD patterns indicated that the synthesized CuO NPs are highly crystalline with monoclinic structure of $\mathrm{CuO}$, which was confirmed by the Joint Committee on Powder Diffraction Standards (JCPDS) (Card No.: 89-5895).

The XRD pattern of Bacillus ef1 revealed the orientation and crystalline nature of $\mathrm{CuO}$ NPs. The peak position with $2 \theta$ values of $32.41^{\circ}, 35.39^{\circ}, 38.86^{\circ}, 48.67^{\circ}, 53.40^{\circ}, 58.28^{\circ}, 61.43^{\circ}$, $65.69^{\circ}, 68.18^{\circ}, 72.37^{\circ}$ and $75.10^{\circ}$ are indexed as (110), (111), (111), (202), (020), (202), (113), (311), (220), (220), and (004) planes, which are in good agreement with those of powder $\mathrm{CuO}$ obtained from the International Center of Diffraction Data card (JCPDS-80-1916) confirming the formation of a crystalline monoclinic structure. No extra diffraction peaks of other phases are detected, indicating the phase purity of $\mathrm{CuO}$ NPs.

\section{References}

1. Grigore, M.E.; Biscu, E.R.; Holban, A.M.; Gestal, M.C.; Grumezescu, A.M. Methods of synthesis, properties and biomedical applications of $\mathrm{CuO}$ Nanoparticles. Pharmaceuticals 2016, 9, 75. [CrossRef]

2. Katwal, R.; Kaur, H.; Sharma, G.; Naushad, M.; Pathania, D. Electrochemical synthesized copper oxide Nanoparticles for enhanced photocatalytic and antimicrobial activity. J. Ind. Eng. Chem. 2015, 31, 173-184. [CrossRef]

3. Sastry, M.; Ahmad, A.; Khan, M.I.; Kumar, R. Biosynthesis of metal Nanoparticles using fungi and actinomycetes. Curr. Sci. 2003, 85, 162-170.

4. Mandal, D.; Bolander, M.E.; Mukhopadhyay, D.; Sarkar, G.; Mukherjee, P. The use of microorganisms for the formation of metal nanoparticles and their application. Appl. Microbiol. Biotechnol. 2006, 69, 485-492. [CrossRef]

5. Rahmatolahzadeh, R.; Aliabadi, M.; Motevalli, K. Cu and $\mathrm{CuO}$ nanostructures: Facile hydrothermal synthesis, characterization and photocatalytic activity using new starting reagents. J. Mater. Sci. Mater. Electron. 2017, 28, 148-156. [CrossRef]

6. Sagadevan, S.; Pal, K.; Chowdhury, Z.Z. Fabrication of $\mathrm{CuO}$ nanoparticles for structural, optical and dielectric analysis using chemical precipitation method. J. Mater. Sci. Mater. Electron. 2017, 28, 12591-12597. [CrossRef]

7. Zayyoun, N.; Bahmad, L.; Laânab, L.; Jaber, B. The effect of $\mathrm{pH}$ on the synthesis of stable $\mathrm{Cu}_{2} \mathrm{O} / \mathrm{CuO}$ Nanoparticles by sol-gel method in a glycolic medium. Appl. Phys. A 2016, 122, 488. [CrossRef]

8. Shi, G.; Bao, Y.; Chen, B.; Xu, J. Phenol hydroxylation over cubic/monoclinic mixed phase CuO Nanoparticles prepared by chemical vapor deposition. React. Kinet. Mech. Cat. 2017, 122, 289-303. [CrossRef]

9. Zhang, Q.; Zhang, K.; Xu, D.; Yang, G.; Huang, H.; Nie, F.; Liu, C.; Yang, S. CuO nanostructures: Synthesis, characterization, growth mechanisms, fundamental properties, and applications. Progress Mater. Sci. 2014, 60, 208-337. [CrossRef]

10. Waris, A.; Din, M.; Ali, A.; Ali, M.; Afridi, S.; Baset, A.; Khan, A.U. A comprehensive review of green synthesis of copper oxide nanoparticles and their diverse biomedical applications. Inorgan. Chem. Commun. 2021, 123, 1387-7003. [CrossRef]

11. $\mathrm{Wu}$, F.; Harper, B.J.; Crandon, L.E.; Harper, S.L. Assessment of $\mathrm{Cu}$ and $\mathrm{CuO}$ nanoparticle ecological responses using laboratory small-scale microcosms Environ. Sci. Nano 2020, 7, 105-115. [CrossRef] [PubMed]

12. Lontie, R. Copper Proteins and Copper Enzymes; CRC Press: Boca Raton, FL, USA, 1984.

13. Kimura, T.; Nishioka, H. Intracellular generation of superoxide by copper sulphate in Escherichia coli. Mutat. Res. 1997, 389, 237-242. [CrossRef]

14. Nies, D.H. Microbial heavy-metal resistance. Appl. Microbiol. Biotechnol. 1999, 51, 730-750. [CrossRef] 
15. Ramasamy, K.P.; Rajasabapathy, R.; Lips, I.; Mohandass, C.; James, R.A. Genomic features and copper biosorption potential of a new Alcanivorax sp. VBW004 isolated from the shallow hydrothermal vent (Azores, Portugal). Genomics 2020, 112, $3268-3273$. [CrossRef] [PubMed]

16. Pucciarelli, S.; Devaraj, R.R.; Mancini, A.; Ballarini, P.; Castelli, M.; Schrallhammer, M.; Petroni, G.; Miceli, C. Microbial consortium associated with the Antarctic marine ciliate Euplotes focardii: An investigation from genomic sequences. Microb. Ecol. 2015, 70, 484-497. [CrossRef] [PubMed]

17. Pucciarelli, S.; La Terza, A.; Ballarini, P.; Barchetta, S.; Yu, T.; Marziale, F.; Passini, V.; Methé, B.; Detrich, H.W., 3rd; Miceli, C. Molecular cold-adaptation of protein function and gene regulation: The case for comparative genomic analyses in marine ciliated protozoa. Mar. Genomics 2009, 2, 57-66. [CrossRef]

18. John, M.S.; Nagoth, J.A.; Ramasamy, K.P.; Ballarini, P.; Mozzicafreddo, M.; Mancini, A.; Telatin, A.; Liò, P.; Giuli, G.; Natalello, A.; et al. Horizontal gene transfer and silver nanoparticles production in a new Marinomonas strain isolated from the Antarctic psychrophilic ciliate Euplotes focardii. Sci. Rep. 2020, 10, 1-14. [CrossRef]

19. John, M.S.; Nagoth, J.A.; Ramasamy, K.P.; Mancini, A.; Giuli, G.; Natalello, A.; Ballarini, P.; Miceli, C.; Pucciarelli, S. Synthesis of bioactive silver nanoparticles by a pseudomonas strain associated with the Antarctic psychrophilic protozoon Euplotes focardii. Mar. Drugs 2020, 18, 38. [CrossRef]

20. Orlando, M.; Pucciarelli, S.; Lotti, M. Endolysins from Antarctic Pseudomonas Display lysozyme activity at low temperature. Mar. Drugs. 2020, 18, 579. [CrossRef]

21. Ramasamy, K.P.; Telatin, A.; Mozzicafreddo, M.; Miceli, C.; Pucciarelli, S. Draft genome sequence of a new pseudomonas sp. Strain, ef1, Associated with the psychrophilic Antarctic ciliate Euplotes focardii. Microbiol. Resour. Announ. 2019, 8, e00867-19. [CrossRef]

22. Chu, W.L.; Dang, N.L.; Kok, Y.Y.; Yap, K.S.I.; Phang, S.M.; Convey, P. Heavy metal pollution in Antarctica and its potential impacts on algae. Polar Sci. 2019, 20, 75-83. [CrossRef]

23. Barker, P.F.; Thomas, E. Origin, signature and palaeoclimatic influence of the antarctic circumpolar current. Earth-Sci. Rev. 2004, 66, 143-162. [CrossRef]

24. Bargagli, R. Environmental contamination in Antarctic ecosystems. Sci. Total Environ. 2008, 400, 212-226. [CrossRef]

25. De Souza, M.J.; Nair, S.; Loka Bharathi, P.A.; Chandramohan, D. Metal and antibiotic-resistance in psychrotrophic bacteria from Antarctic Marine waters. Ecotoxicology 2006, 15, 379-384. [CrossRef]

26. Lo Giudice, A.; Casella, P.; Bruni, V.; Michaud, L. Response of bacterial isolates from Antarctic shallow sediments towards heavy metals, antibiotics and polychlorinated biphenyls. Ecotoxicology 2013, 22, 240-250. [CrossRef]

27. Mancini, A.; Pucciarelli, S. Antibiotic activity of the antioxidant drink effective Microorganism-X (EM-X) extracts against common nosocomial pathogens: An in vitro study. Nat. Prod. Res. 2018, 4, 1-6. [CrossRef]

28. Ramanathan, R.; Field, M.R.; O’Mullane, A.P.; Smooker, P.M.; Bhargava, S.K.; Bansal, V. Aqueous phase synthesis of copper nanoparticles: A link between heavy metal resistance and nanoparticle synthesis ability in bacterial systems. Nanoscale 2013, 5 , 2300-2306. [CrossRef] [PubMed]

29. Honary, S.; Barabadi, H.; Ghara-El Fathabad, B.; Naguib, F. Green synthesis of copper oxide Nanoparticles using Penicillium aurantiogriseum, Penicillium citrinum and Penicillium waksmanii. Digest. J. Nanomat. Bios. 2012, 7, 999-1005.

30. Yedurkar, S.M.; Maurya, C.B.; Mahanwar, P.A. A biological approach for the synthesis of copper oxide nanoparticles by Ixora Coccinea leaf extract. J. Mat. Environ. Sci. 2017, 8, 1173-1178.

31. Dhineshbabu, N.R.; Rajendran, V.; Nithyavathy, N.; Vetumperumal, R. Study of structural and optical properties of cupric oxide nanoparticles. Appl. Nanosci. 2016, 6, 933-939. [CrossRef]

32. Jovanović, Ž.; Radosavljević, A.; Šiljegović, M.; Bibić, N.; Mišković-Stanković, V.; Kačarević-Popović, Z. Structural and optical characteristics of silver/poly(N-vinyl-2-pyrrolidone) nanosystems synthesized by $\gamma$-irradiation. Radiat. Phys. Chem. 2012, 81, 1720-1728. [CrossRef]

33. Datta Majumdar, T.; Singh, M.; Thapa, M.; Dutta, M.; Mukherjee, A.; Ghosh, C.K. Size-dependent antibacterial activity of copper nanoparticles against Xanthomonas oryzae pv. oryzae-A synthetic and mechanistic approach. Colloid Interface Sci. Commun. 2019, 32, 100190. [CrossRef]

34. Dadi, R.; Azouani, R.; Traore, M.; Mielcarek, C.; Kanaev, A. Antibacterial activity of ZnO and CuO nanoparticles against gram positive and gram negative strains. Mater. Sci. Eng. C 2019, 104, 109968. [CrossRef]

35. Ramzan, M.; Obodo, R.M.; Mukhtar, S.; Ilyas, S.Z.; Aziz, F.; Thovhogi, N. Green synthesis of copper oxide nanoparticles using Cedrus deodara aqueous extract for antibacterial activity. Mater. Today Proc. 2021, 36, 576-581. [CrossRef]

36. Applerot, G.; Lellouche, J.; Lipovsky, A.; Nitzan, Y.; Lubart, R.; Gedanken, A.; Banin, E. Understanding the antibacterial mechanism of $\mathrm{CuO}$ nanoparticles: Revealing the route of induced oxidative stress. Small 2012, 8, 3326-3337. [CrossRef] [PubMed]

37. Shuai, C.; Guo, W.; Gao, C.; Yang, Y.; Wu, P.; Feng, P. An nMgO containing scaffold: Antibacterial activity, degradation properties and cell responses. Int. J. Bioprint. 2018, 4, 120. [CrossRef] [PubMed]

38. CLSI Document EP100-S23; Clinical and Laboratory Standards Institute: Wayne, PA, USA, 2019. 